\title{
ARDUINO ATMEGA-328 MICROCONTROLLER
}

\section{R.HARI SUDHAN ${ }^{1}$ M.GANESH KUMAR ${ }^{2}$ A.UDHAYA PRAKASH ${ }^{3}$ S.ANU ROOPA DEVI ${ }^{4}$ P. SATHIYA $^{5}$}

Student, Instrumentation and Control Engineering, Saranathan College of Engineering, Trichy, India ${ }^{1,2,3,4}$

Assistant Professor, Instrumentation and Control Engineering, Saranathan College of Engineering, Trichy, India ${ }^{5}$.

\begin{abstract}
Arduino ATMEGA-328 microcontroller has been programmed for various applications. By using the power jack cable, arduino microcontroller has been programmed so that the execution of the program may takes place. Various kinds of arduino board are present in the market. In this paper, Arduino UNO ATMEGA-328 microcontroller is described in a detailed manner. Arduino software is installed in the computer and so that we can edit and upload the program according to the applications. Mainly these arduino software supports $\mathrm{c}$ and c++ programming languages. Various inputs and outputs are present in the arduino board and therefore simultaneously 8 input and output ports can be used for various applications. Some of the applications used by using arduino boards are rotating general motor, stepper motor, control valve open, etc.,
\end{abstract}

KEYWORDS: ATMEGA 328, Aurdino, computer applications, Microcontroller

\section{INTRODUCTION}

Nearly 7, 00,000 numbers of arduinos are present in the are high level languages. Normally it has 18 number of market. Of these, Arduino ATMEGA-328 microcontroller input and output pins.

consist of 14 input and output analog and digital pins (from this 6 pins are considered to be a PWM pins), 6 analog inputs and remaining digital inputs. Power jack cable is used to connect arduino board with the computer. Externally battery is connected with the arduino microcontroller for the power supply. Arduino is an open source microcontroller from which there is no feedback present in the microcontroller. This arduino board consist of $\mathrm{I} 2 \mathrm{C}$ bus, that can be able to transfer the data from arduino board to the output devices. These arduino boards are programmed over RS232 serial interface connections with atmega arduino microcontrollers. The operating volt ranges from $5 \mathrm{v}$. The input voltage recommended for arduino microcontroller is from $7 \mathrm{v}$ and the maximum of $12 \mathrm{v}$. The DC input current given to the arduino board is in the range of $40 \mathrm{~mA}$.

It consists of different types of memories such as flash memory, EEPROM, SRAM. The length of the arduino board is nearly about $68.64 \mathrm{~mm}$ and the width of the microcontroller is about $53.4 \mathrm{~mm}$. The weight of the arduino microcontroller is about $20 \mathrm{~g}$. We can use various types of microcontroller such as 8 bit AVL Atmel microcontroller and 32 bit Atmel arm microprocessor. From these different kinds of processors, we can use those processors for various engineering projects as well as industrial applications. Some of the examples of using the arduino in the industrial applications are controlling the actuators and sensors. Some of the examples of arduino microcontrollers are Arduino Duemilanove, Arduino UNO, Arduino Leonardo, Arduino Mega, Arduino MEGA 2560 R3, Arduino MEGA 2560 R3, Arduino Nano, Arduino Due, LilyPad Arduino, micro arduino. We have already mentioned, arduino has been programmed by using $\mathrm{c}$ and $\mathrm{c}++$ programming language. These $\mathrm{c}$ and $\mathrm{c}++$
Among those 6 pins are considered to be an analog inputs. From these analog inputs, we can be able to work the arduino microcontroller using analog inputs supply. Normally analog inputs can be in the range of $0-5 \mathrm{~V}$. Similar to that digital inputs are present in the microcontroller which can act the use of microcontroller using digital inputs. Digital inputs can be in the range of $5 \mathrm{~V}$.

ATMEGA 328 microcontroller, which acts as a processor for the arduino board. Nearly it consists of 28 pins. From these 28 pins, the inputs can be controlled by transmitting and receiving the inputs to the external device. It also consists of pulse width modulation (PWM). These PWM are used to transmit the entire signal in a pulse modulation. Input power supply such as Vcc and Gnd are used. These IC mainly consists of analog and digital inputs. These analog and digital inputs are used for the process of certain applications.

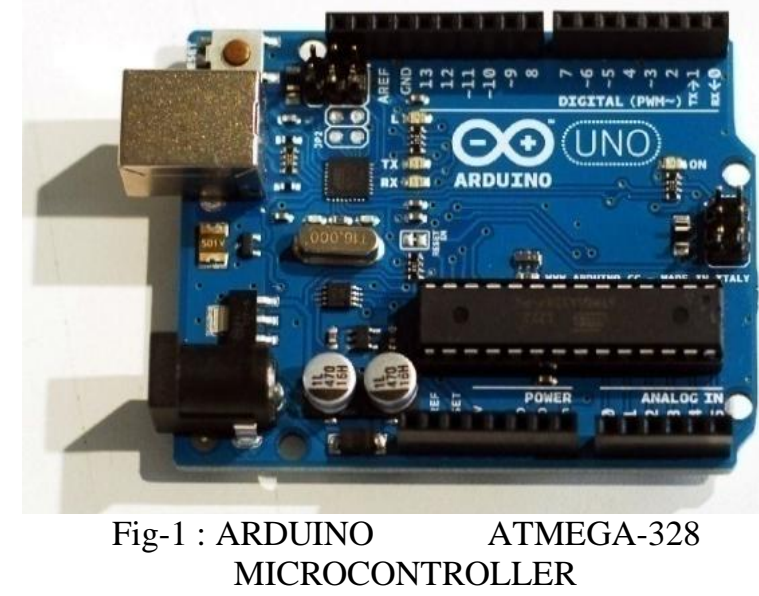




\section{DESCRIPTION}

\section{- INPUT:}

\section{ANALOG INPUT:}

Arduino atmega-328 microcontroller board consist of 6 analog inputs pins. These analog inputs can be named from A0 to A5. From these 6 analog inputs pins, we can do the process by using analog inputs. Analog inputs can be used in the operating range of 0 to $5 \mathrm{~V}$.

Analog signal is considered as the continuous time signal, from which these analog signal can be used for certain applications. These are also called as non discrete time signal.

Inputs such as voltage, current etc.., are considered to be either analog signal or digital signal only by analysing the time signal properties. Various applications of arduino microcontroller can use only an analog input instead of digital inputs. For these applications, analog input ports or pins can be used.

\section{DIGITAL INPUT:}

Digital inputs can be defined as the non continuous time signal with discrete input pulses. It can be represented as 0 's and 1's. These digital inputs can be either on state or in off state. Arduino atmega328 microcontroller also consists of 12 digital input pins. It can be stated as D0 to D11. Nearly 12 inputs can be used for digital input/output applications.

The working of the digital input ports is where the discrete input pulses can be triggered and supplied to the ports. These ports receive the input and therefore the port can be used for both input and output process. These digital pins can access only the digital inputs.

\section{ATMEGA-328 IC:}

This ATMEGA-328 integrated chip consists of 28 pins. It consists of 6 analog inputs that are shown in the pin diagram. Analog inputs can be represented as PC0 to PC5. These analog input pins posses the continuous time signal which acts as an analog input for the system. Further it also consists of 12 digital inputs.

It can be represented as PD1 to PD11 which act as an digital input ports based on pulse width modulation (PWM). These PWM, which transmits the signal in the form of discredited form. Both analog and digital input ports can be used for various applications for the input power supply, VCC and GND pins are used. Pins PB6 and PB7, which acts as a crystal to generate a clock signal. By using these crystal, we can generate the clock signals and by these clock signals, we can use this clock signals for input sources.

PC6 pin are the one where it can be used for the reset option. Resetting the program can be done by using this PC6 pin.

The pin diagram of atmega-328 microcontroller can be shown below.

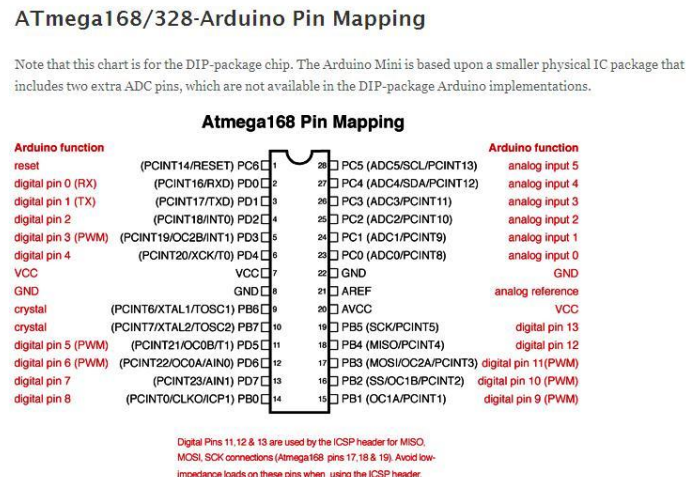

Fig-2 : Pin Configuration

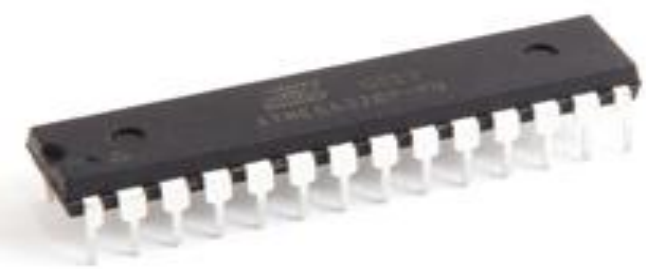

Fig-3 ATMEGA-328 IC

\section{POWER JACK CABLE / USB PORT:}

This Arduino atmega-328 microcontroller can be interfaced with the other electronic devices such as computer by using USB port or power jack cable from these power jack cable, we can upload the program of Arduino for their applications. At first, the program can be initialised or can be edited by using Arduino software tools. Then these programs can be transferred through arduino microcontroller board by using usb cable or power jack cable.

\section{- POWER SUPPLY:}

There is an additional power supply source present in Arduino microcontroller. Power supply port is present at the corner of the arduino microcontroller. Either we can use this power supply port by connecting with external power supply.(ie, ac power supply), or by connecting an dc power supply through input pins. These power supplies produce an active form to the arduino microcontroller. These arduino microcontrollers can accept a range of power supply. When the power supply voltage range exceeds, the microcontroller gets damaged. Hence, only the particular range of power supply should be given to the arduino microcontroller.

\section{WORKING PRINCIPLE}

The working of arduino microcontroller is where the proper connection is made. Checking all the input ports as well as the power supply connection. The output of the pins can be connected with the external devices according to their applications. The program to be executed for the applications can be done by using arduino software. From this arduino software, we can edit according to the applications. This software can works on $\mathrm{c}$ and c++ programming language. It is fully a high level language. By using the conditions of working, we can create a 
program to proceed for the applications. Then after, these clockwise or an anticlockwise direction. By using these programs can be uploaded through the arduino Arduino microcontrollers, we can controller speed, microcontroller by using the power jack cable. The direction and also the number of steps of a motor. program can be uploaded to the microcontroller and ready Likewise these arduino microcontrollers can be used for for further process. ATMEGA-328 microcontroller can many numbers of applications.

saves a program and these IC can acts as a processor to do the process without any error. After by giving an analog or digital input to the system, we can do the process according to the applications. We can control the process of the application by editing the program in the arduino software and again can be uploaded to the arduino microcontroller via power jack cable. There is an option of reset button. The purpose of reset button is to reset the program which means the previous programs are deleted and we can use the arduino for the other application purposes. Likewise, these arduino ATMEGA-328 microcontrollers can be used for $\mathrm{n}$ number of applications. These arduino microcontrollers are widely used in automation industries for controlling the process and to work the system in an automation mode.

Here, I have provided a simple arduino program to do the process of rotating a stepper motor for one revolution. There are many number of example programs that are present in the arduino software. We can edit these programs for our applications purposes. The example program can be given below.

\section{PROGRAM}

Stepper Motor Control - one revolution This program drives a unipolar or bipolar stepper motor. The motor is attached to digital pins $8-11$ of the Arduino. The motor should revolve one revolution in one direction, then one revolution in the other direction.

\#include < Stepper.h> const int steps Per Revolution = 200; $/ /$ change this to fit the number of steps per revolution for your motor

// initialize the stepper library on pins 8 through 11: Stepper my Stepper (steps Per Revolution, 8,9,10,11); void $\operatorname{setup}()$

// set the speed at 60 rpm: my Stepper. Set Speed(60);

// initialize the serial port: Serial. begin(9600);

Void $\operatorname{loop}()$

// step one revolution in one direction: Serial. println("clockwise"); my Stepper. step(steps Per Revolution);delay(500);

// step one revolution in the other direction: Serial. println("counter clockwise"); my Stepper. Step (steps Per Revolution); delay (500); \}

From the above program, the Arduino can produce a digital input signal to the stepper motor. The stepper motor which in turn receives the signal from the arduino microcontroller and therefore it can rotate either in 